\title{
Archéopages
}

Archéopages

Archéologie et société

42 | 04-07/2015

Construire en terre crue

\section{La construction en terre crue de l'âge du Fer à nos jours}

L'apport de la micromorphologie à la compréhension des techniques

Earthen architecture from the Iron Age to the present day. The contribution of micromorphology to the understanding of techniques

La construcción de barro, desde la Edad del Hierro hasta nuestros días. El aporte de la micromorfología a la comprensión de las técnicas

\section{Cécilia Cammas}

\section{OpenEdition}

Journals

Édition électronique

URL : https://journals.openedition.org/archeopages/1208

DOI : 10.4000/archeopages. 1208

ISSN : 2269-9872

Éditeur

INRAP - Institut national de recherches archéologiques préventives

Édition imprimée

Date de publication : 1 mars 2016

Pagination : 58-67

ISSN : 1622-8545

Référence électronique

Cécilia Cammas, "La construction en terre crue de l'âge du Fer à nos jours », Archéopages [En ligne], 42 | 04-07/2015, mis en ligne le 01 juillet 2017, consulté le 02 juin 2021. URL : http://

journals.openedition.org/archeopages/1208; DOI : https://doi.org/10.4000/archeopages. 1208 


\section{La construction en terre crue \\ de l'âge du Fer à nos jours L'apport de la micromorphologie à la compréhension des techniques}

Cécilia Cammas Inrap, UMR 5140 «Archéologie des sociétés méditerranéennes»

58

Létude de la terre crue est riche d'implications et peut documenter différents champs de la recherche archéologique. La nature même du matériau terre et le début de la chaîne opératoire (choix des sédiments, prélèvement) témoignent de la connaissance du milieu et des savoirs, souvent empiriques, des propriétés des différentes terres. Létude des constructions en terre crue est, par conséquent, une manière d'approcher l'interaction entre l'homme et son milieu. L'étude des élévations et des restes de matériaux de construction en terre crue est quant à elle une source de renseignement sur la nature et l'évolution du cadre et des modes de vie des populations passées.

L'objectif de cet article est de présenter la démarche géoarchéologique spécifique d'étude des matériaux de construction en terre crue développée sur un large corpus de sites et de matériaux prélevés dans des contextes géographiques et chronologiques très variés, afin de proposer des clés de détermination des différentes techniques, opérationnelles dans les situations où les matériaux et les techniques sont moins bien connus, ou dans celles où les vestiges sont tellement altérés que leur identification est difficile sur le terrain.

Un matériau de construction largement diffusé mais difficile à appréhender

La terre est un matériau de construction utilisé depuis les périodes les plus anciennes, dans une large aire géographique (Chazelles, 1997). Dans l'espace français, dès le Néolithique, les techniques de construction en terre crue semblent déjà largement diffusées aussi bien dans le Nord que dans le Sud (Wattez, 2003, 2009; Onfray, 2014, voir les contributions sur le Néolithique dans ce volume). En ce qui concerne les périodes protohistoriques et historiques, il existe une diversité d'usage de la terre. Pour les murs, les principaux modes de mise en œuvre de la terre crue connus d'après les textes et les données de l'archéologie sont la bauge (ou murs modelés), le pisé (terre compactée et damée dans un coffrage en bois), la brique crue moulée ou non et le torchis (terre appliquée sur une armature porteuse en végétaux). La terre est aussi utilisée pour confectionner les sols, par exemple les sols en «terre battue».

La terre - ou plutôt les terres devrait-on dire au vu de la diversité observée - présente la particularité d'être un matériau facilement accessible, souple et malléable. Il est facile de créer une ouverture ou de la boucher pour adapter son environnement construit à de nouveaux besoins. L'entretien et les réfections régulières nécessaires à la pérennisation des constructions en terre sont également autant d'occasions d'améliorer ou de modifier l'apparence ou la structure du bâti. De ce fait, les constructions en terre crue, et plus particulièrement les maisons d'habitation, peuvent facilement évoluer au cours du temps, sur des périodes courtes, à l'échelle d'une ou plusieurs générations.

La construction en terre crue d'ensembles bâtis de grande envergure, tels que des groupes de maisons, des villages, ou des quartiers entiers de villes, implique une infrastructure pour l'extraction, la transformation et le transport des matériaux, ainsi que, par exemple, pour le moulage des briques crues. Il est alors possible d'aborder la question de la standardisation des techniques et de la présence de groupes de la population, les artisans, qui produisent et mettent en œuvre ces matériaux. L'identification et la caractérisation des techniques documentent leur apparition et leur diffusion géographique et culturelle. 


\begin{tabular}{|c|c|c|}
\hline Site & Périodes & Type de site \\
\hline Lattes & Âge du Fer - Époque romaine & $\begin{array}{l}\text { Urbain } \\
\text { Murs et sols en terre }\end{array}$ \\
\hline $\begin{array}{l}\text { Littré, Hôtel-Dieu } \\
\text { Bourges (Cher) }\end{array}$ & Limite Premier et second âge du Fer & Urbain \\
\hline $\begin{array}{l}\text { Paris, matériaux provenant de } 12 \text { sites archéo- } \\
\text { logiques }\end{array}$ & Époque romaine & Urbain \\
\hline Rue Saint-Brice, Chartres & Époque romaine & Urbain, caves \\
\hline Mours (Val-d'Oise) & Époque romaine & Élévations en briques crues d'un four de tuilier \\
\hline Aspiran (Hérault) & Époque romaine & $\begin{array}{l}\text { Rural } \\
\text { Habitat }\end{array}$ \\
\hline Port-la-Nouvelle (Aude) & Époque romaine & Habitat \\
\hline Mouriès (Bouches-du-Rhône) & Époque romaine & Habitat \\
\hline $\begin{array}{l}4 \text { rue Brûlée } \\
\text { Strasbourg (Bas-Rhin) }\end{array}$ & Époque romaine et $\mathrm{x}^{\mathrm{e}}-\mathrm{XI}^{\mathrm{e}}$ siècle & Urbain \\
\hline $\begin{array}{l}\text { Les Baudets / Bronville, Le Boullay-Thierry } \\
\text { (Eure-et-Loir) }\end{array}$ & $\begin{array}{l}\text { Époque romaine } \\
\text { Époque mérovingienne }\end{array}$ & $\begin{array}{l}\text { Urbain } \\
\text { Bases de murs }\end{array}$ \\
\hline Aimargues (Gard) & Premier Moyen Âge & $\begin{array}{l}\text { Village } \\
\text { Bases de murs }\end{array}$ \\
\hline Clos des Forges, Avrilly (Eure) & Époque médiévale & $\begin{array}{l}\text { Village médiéval } \\
\text { Zone artisanale }\end{array}$ \\
\hline $\begin{array}{l}\text { Fort-Saint-Sébastien, Saint-Germain-en-Laye } \\
\text { (Yvelines) }\end{array}$ & 1669-1671 (remblaiement du site) & Camp d'entraînement des troupes de Louis XIV \\
\hline $\begin{array}{l}\text { Sainte-Christie d'Armagnac (Gers) Juilles (Gers) } \\
\text { et Fontignan-Savès (Haute-Garonne) }\end{array}$ & Époque moderne & $\begin{array}{l}\text { Archéologie du bâti } \\
\text { Édifices en élévation }\end{array}$ \\
\hline Ampurias (Espagne) & Époque romaine & Habitat, mur \\
\hline $\begin{array}{l}\text { La Rábita de Guardamer del Segura (province } \\
\text { d'Alicante, Espagne) }\end{array}$ & Bronze / Fer $(725 / 500 \mathrm{BC})$ & Habitat \\
\hline Shabwa, Hadhramawt (Yémen) & Fin second millénaire - viII ${ }^{e}$ siècle $B C$ & Habitat, murs \\
\hline Bat (Sultanat d'Oman) & Âge du Bronze & Habitat murs, sols \\
\hline $\begin{array}{l}\text { Gongying, Municipalité de Nanyang, Province } \\
\text { du Henan, (République populaire de Chine) }\end{array}$ & Âge du Bronze $\mathrm{x}^{\mathrm{e}} \mathrm{s}-\mathrm{vi}^{\mathrm{e}} \mathrm{s}$ BC Fluviosols & Habitat, sols \\
\hline Rihra, Sidi Slimane (Maroc) & $\begin{array}{l}\text { Tell de l'âge du Fer } \\
\text { Époque romaine }\end{array}$ & Habitat, murs, sols \\
\hline
\end{tabular}

\section{Liste des principaux sites}

ayant fourni des matériaux

de construction en terre

crue, et qui constituent des

éléments importants du

corpus d'étude ; les sites

sont implantés dans des

contextes climatiques

(continental,

méditerranéen, semi-aride

à aride), géographiques et

chrono-culturels différents. 


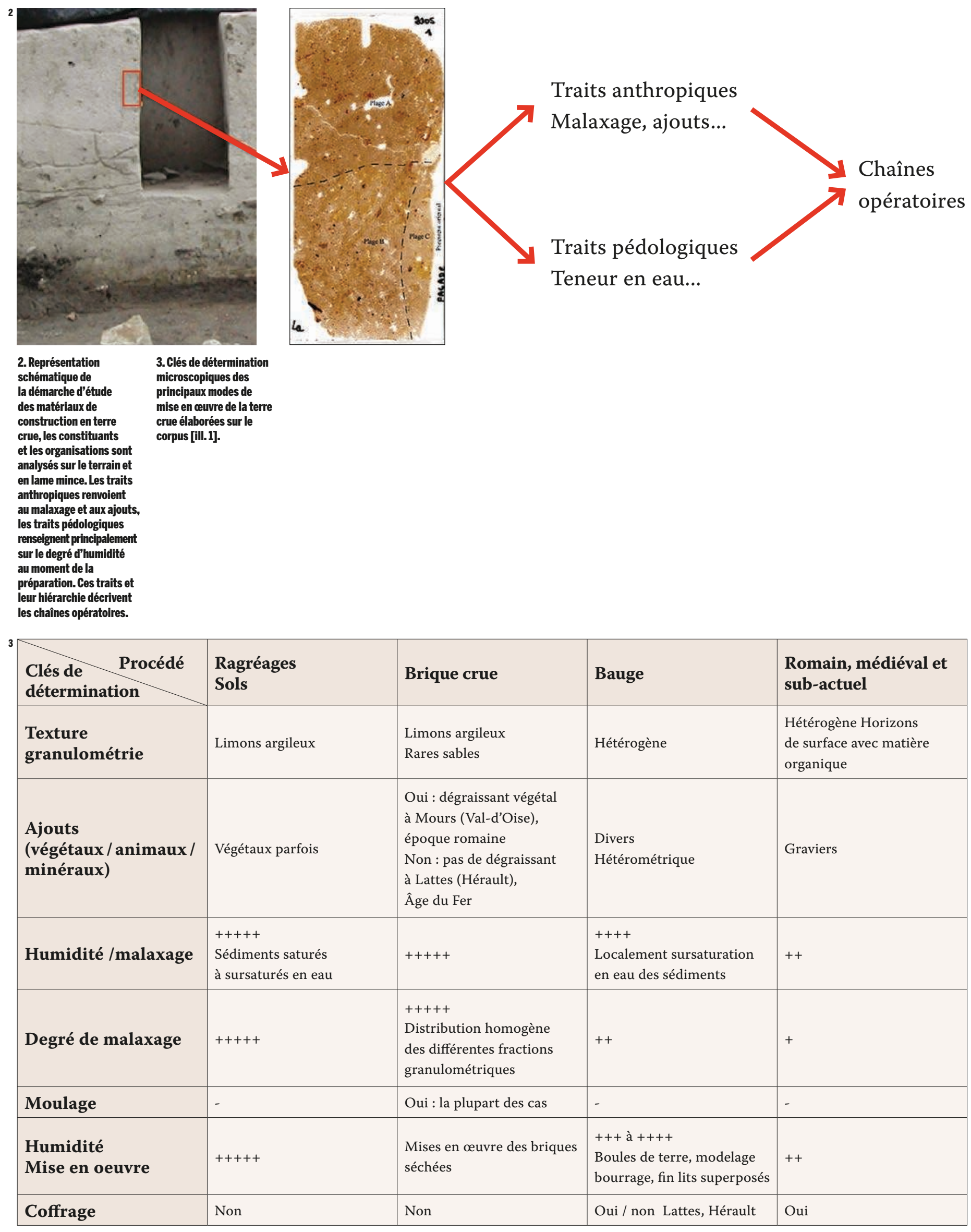


Cependant, en archéologie, la construction et les matériaux de construction en terre crue sont identifiés et étudiés dès la phase de terrain de manière quasi systématique dans le sud de la France, alors que dans la France septentrionale, on constate un déficit d'intérêt et d'étude. En outre, trop souvent, dans les rapports et les publications archéologiques, l'utilisation des termes «bauge» ou « pisé », qui recouvrent des techniques ayant une histoire totalement différente, sont utilisés de manière indifférenciée. La période d'apparition du pisé faisant actuellement l'objet de recherches, l'identification correcte des techniques prend toute son importance. Tous ces travaux reposent sur les données issues de la caractérisation des différentes terres employées dans la construction ainsi que celle des transformations qu'elles ont subies, c'està-dire les chaînes opératoires.

Létude archéologique et macroscopique minutieuse des restes de matériaux de construction en terre crue en élévation, ou en position secondaire, est bien sûr indispensable pour identifier les matériaux et les techniques (Chazelles, 1997 ; Cammas et Chazelles, 2013). Cependant, sur le terrain, la nature des matériaux et les techniques utilisées sont souvent difficiles à préciser, et la différenciation entre le pisé et la bauge, en particulier, pose problème. Parmi les méthodes d'étude de la terre crue, la géoarchéologie et la micromorphologie ont été utilisées depuis longtemps pour caractériser les matériaux de construction en terre crue sur les sites archéologiques et comprendre leur mode de dégradation (Courty et al., 1989 ; Gé et al., 1993 Rosen, 1993 ; Matthews, 1995 ; Goldberg et Macphail, 2006 ; Goodman-Elgar, 2008 ; Friesem et al., 2014), mais une approche micromorphologique plus originale a été développée au cours des années 1990 et 2000 sur des sites protohistoriques et historiques du sud et du nord de la France (Cammas, 1994, 1999, 2003 ; Roux et Cammas, 2007, 2010 ; Duvernay, 2003). Celle-ci vise à restituer les chaînes opératoires à l'aide de la micromorphologie afin d'identifier les techniques passées (Cammas, 2003).

\section{Un corpus riche et une méthodologie adaptée}

Létude des matériaux de construction présentée ici s'est fondée d'abord sur l'analyse d'un corpus provenant dans sa grande majorité du site de Lattes (Hérault, $\mathrm{IV}^{\mathrm{e}}$ siècle avant notre ère- $\mathrm{II}^{\mathrm{e}}$ siècle de notre ère) (Cammas, 1994, 1999), étudié et analysé depuis les années 1990 dans le cadre des fouilles ${ }^{1}$. En parallèle, le corpus s'est enrichi d'échantillons collectés par le réseau constitué à l'UMR Archéologie des sociétés méditerranéennes (C.-A. de Chazelles et J.-C. Roux) dans différentes situations géographiques et culturelles, en France et à l'étranger. D'autres échantillons proviennent de différents sites du nord et du sud de la France, ils ont été prélevés lors de fouilles préventives menées par l'Inrap sur des sites s'échelonnant de l'âge du Fer à l'époque moderne [ill. 1]. Des matériaux de construction ont aussi été repérés ponctuellement et étudiés dans de nombreux autres sites archéologiques. Ce corpus est complété, à titre

de comparaison, par des échantillons provenant de constructions sub-actuelles ou actuelles, collectés par le réseau professionnel et scientifique de l'Inrap (pisé de la région lyonnaise, par exemple).

L'apport des sciences de la Terre - et plus particulièrement de la micromorphologie - pour ce qui concerne l'étude des matériaux de construction en domaine tempéré n'est plus à démontrer (Cammas, 1999, 2003 ; Duvernay, 2003 ; Cammas et Wattez, 2009; Wattez, 2003, 2009 ; Onfray, 2014 ; Roux et Cammas, 2010). Les objectifs des travaux micromorphologiques sont d'identifier les techniques de construction afin de documenter l'apparition, la diffusion et la disparition de certaines techniques, de caractériser le fonctionnement, l'entretien et le statut des espaces, et, enfin, de servir de référence pour des contextes moins bien connus ou conservés.

La micromorphologie consiste en l'étude des sols au microscope pétrographique et elle « vise à caractériser les constituants et les organisations du sol à toutes les échelles » (Fedoroff, 1979; Fedoroff et Courty, 1994). Afin de conserver et d'étudier l'organisation originelle des sédiments, des prélèvements sont réalisés en blocs non perturbés sur le terrain, ils sont ensuite indurés à l'aide de résine synthétique, puis une tranche de sédiment est découpée et amincie à l'épaisseur standard de 25 microns sur une lame de verre. Cette épaisseur permet d'identifier les constituants et les organisations du sol à l'aide des manuels de description micromorphologique et pétrographique, ainsi qu'avec le recours aux collections de référence que chaque chercheur se doit de constituer.

La micromorphologie s'inscrit dans le prolongement d'une étude de terrain pour ce qui concerne les couches contenant des restes de terre crue, ou d'une étude macroscopique quand il s'agit d'élévations in situ ou de fragments de matériaux de construction. L'échantillonnage des élévations nécessite une réflexion particulière (Roux et Cammas, 2010). Par exemple, une colonne stratigraphique continue effectuée au cœur du mur permettra d'étudier la variabilité verticale des terres et, éventuellement, des techniques utilisées (Roux et Cammas, 2010). Pour la bauge et le pisé, des prélèvements localisés aux interfaces horizontales ou verticales décelables entre les couches lors de l'étude macroscopique minutieuse offrent la possibilité d'identifier et de caractériser les levées de bauge (Roux et Cammas, 2007) ainsi que les lits et les banchées de pisé. Un prélèvement horizontal effectué perpendiculairement à l'axe du mur pourra permettre de distinguer des traces de coffrage (Roux et Cammas, 2007) ou de traitements de surface tels que le lissage ou l'application d'enduits (Duvernay, 2003 ; Cammas, 2008). En ce qui concerne ces enduits, la
Archéologie des sociétés méditerranéennes. 
4. Site de Mours (Vald'Oise), élévation en brique crue du four de tuilier d'époque romaine. La lame mince (à gauche) recoupe deux briques jaunes et un joint. Sous le microscope (à droite), la texture, limono-argileuse, et les caractères de la masse fine indiquent que les sédiments proviennent d'un luvisol, le sol que l'on trouve dans l'environnement proche. Pour cette brique,

l'absence d'agrégats résiduels non

homogénéisés témoigne d'un malaxage fin.

À la base de la brique,

les vides aplatis (Va)

témoignent de la

réorganisation des sédiments au moment

du tassement de la terre dans des moules. Le joint est sableux.

5. Site de Mours (Val-

d'Oise), élévation en

brique crue du four de

tuilier d'époque romaine.

La lame mince (à gauche)

recoupe deux briques

et un joint, reposant sur

du calcaire. Sous le

microscope (à droite),

la masse fine présente

quelques plages calcaires

grises (Calc). Le mélange

luvisol et calcaire

explique la coloration

plus grise de la brique

inférieure. Le malaxage

est plus grossier. Le joint

est sableux. Un fin lit plus massif, avec des graviers

carbonatés, localisé

au-dessus de l'encaissant,

correspond probablement

à un lit de pose.

6. Site de Mours (Val-

d'Oise), briques grises composant l'élévation en

brique crue du four de

tuilier d'époque romaine.

Ces briques sont de

composition différente

des briques jaunes des

illustrations 4 et 5 . L

masse fine est composée

d'un mélange argiles /

carbonates (photo de

gauche), la morphologie

des imprégnations

ferrugineuses, formées

avant l'extraction des

sédiments, indique qu'il

s'agit très probablement

de colluvions évoluées en

zone humide qui ont étê

utilisées. Elles sont peu

malaxées (plage argileuse

appauvrie en fer résiduelle,

photo de droite).

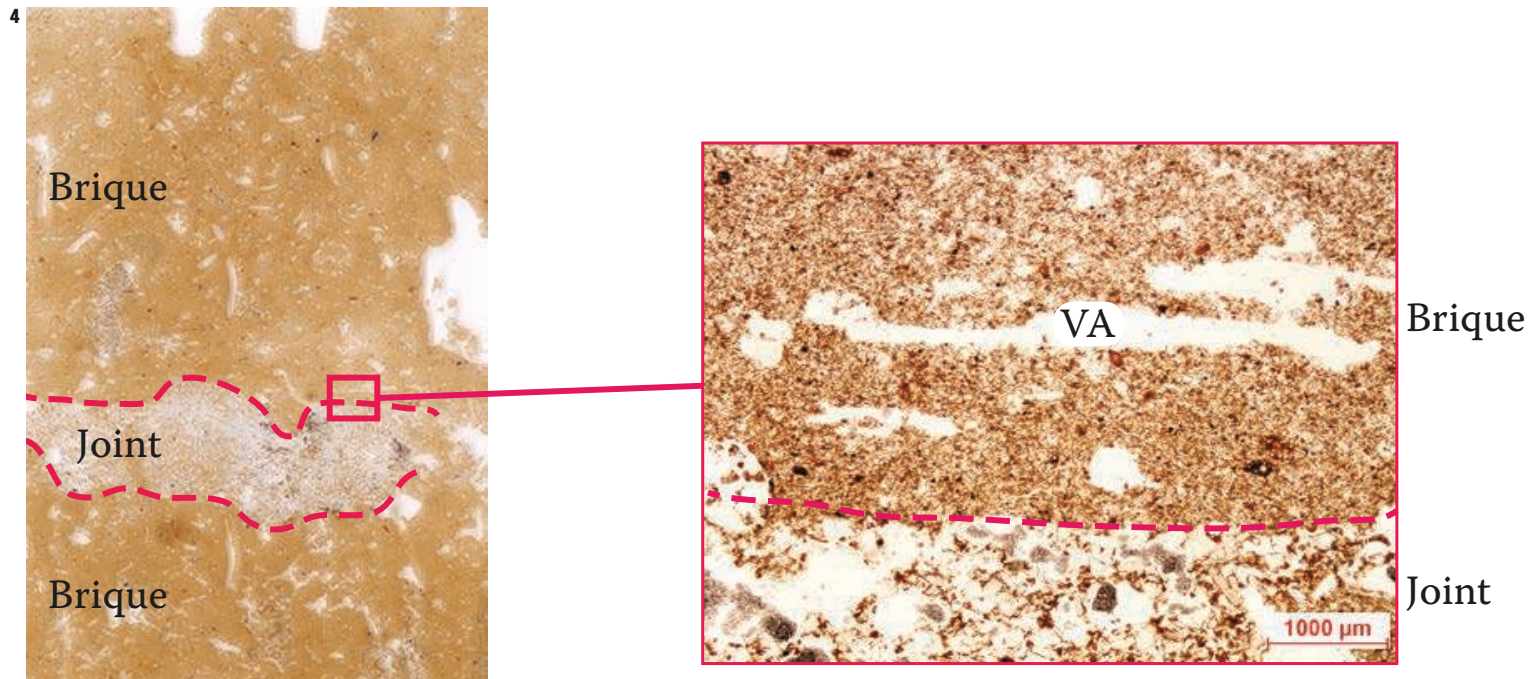

Photo au microscope, lumière polarisée non analysée

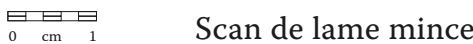

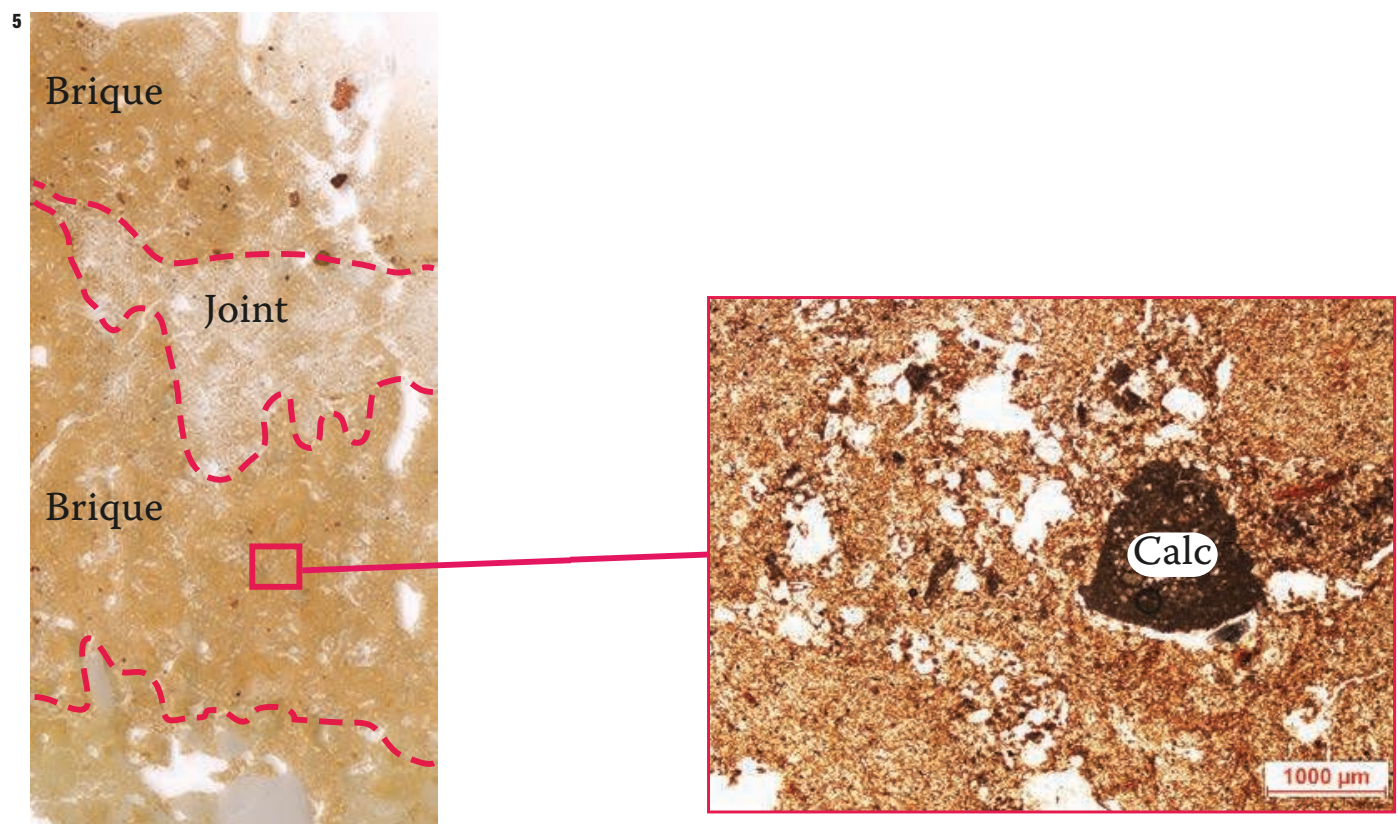

Photo au microscope, lumière polarisée non analysée

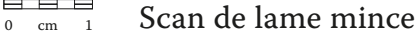
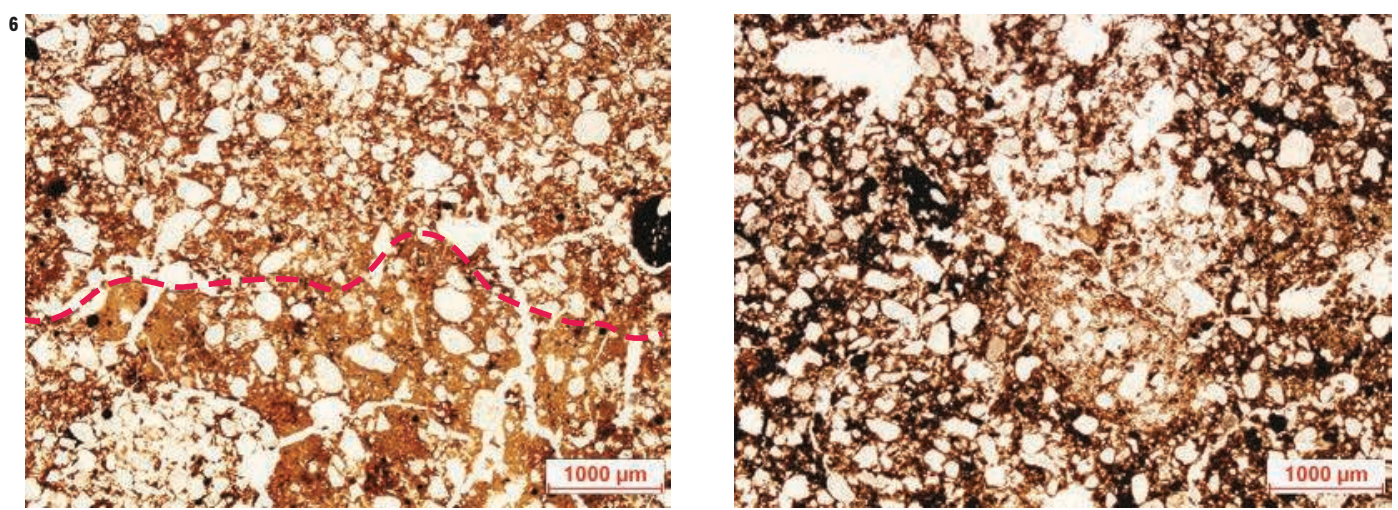

Briques grises 
microstratigraphie qui peut être restituée à l'aide de la micromorphologie rend compte d'une succession de préparations lors d'une seule phase de construction ou de réfection et d'entretien (Cammas, 1994, 1999, 2008, 2010).

Pour le traitement des fragments de matériaux de construction rapportés au laboratoire, une méthode d'étude standardisée a été élaborée (Cammas, 2008). Elle comprend dans un premier temps l'ouverture d'une fiche de description et la photographie de l'échantillon sous toutes ses faces. Ensuite, l'échantillon est scié en deux. Il fait l'objet d'étude sur coupe à la scie et sur cassure fraîche, à l'œil et à la loupe afin d'apprécier la couleur, la texture et l'organisation des sédiments, ainsi qu'une éventuelle stratification. À cette échelle, les inclusions végétales et minérales grossières sont identifiables. Une moitié est conservée comme référence macroscopique ou pour des analyses complémentaires qui pourraient apparaître nécessaires au cours de l'étude. Le second fragment est utilisé pour confectionner une lame mince. L'interprétation finale intègre les résultats des observations macroscopiques et de l'analyse micromorphologique.

L'originalité des recherches présentées ici est de dépasser la simple caractérisation des matériaux de construction identifiés sur le terrain, afin de rechercher des clés de détermination microscopiques permettant de discriminer les différentes techniques, complétant ainsi la vision de terrain. Ces travaux se fondent sur le fait que les processus anthropiques, tels que le malaxage, par exemple, et la proportion d'eau contenue dans les sédiments s'expriment par des caractères morphologiques (ou traits) spécifiques qui peuvent être distingués en lame mince. Il a été possible de lister des traits significatifs, qui nous renseignent sur différents gestes humains correspondant aux étapes de la chaîne opératoire [ill. 2]. Il s'agit de la texture et de la granulométrie, des ajouts végétaux, animaux ou minéraux, du degré d'humidité au moment du malaxage, du degré de malaxage, du moulage, l'humidité des sédiments au moment de la mise en œuvre et la présence éventuelle d'un coffrage [ill. 3]. Cette approche permet d'affiner la connaissance des différents gestes liés à la préparation et à la construction en terre crue, et, à l'échelle microscopique, les chaînes opératoires ainsi repérées correspondent à des techniques différentes (Cammas, 2003). La documentation issue des différents sites constitue des collections de référence de lames minces larges permettant de mettre en évidence des variations chronoculturelles [cf. encadré p. 68].

\section{Les techniques mises en évidence}

Les ragréages, sols d'une épaisseur infracentimétrique, ont été caractérisés d'abord sur le site de Lattes (Hérault). Ils sont confectionnés à partir de limons argileux finement malaxés, ainsi qu'en atteste la faible proportion d'agrégats résiduels. Dans de rares cas, des ajouts végétaux peuvent être observés. Ces sédiments sont épandus à l'état saturé en eau et ils nappent les couches sous-jacentes. Parfois, lors de l'épandage sur des sols rugueux, des bulles d'air aplaties sont emprisonnées à la base de cet apport (Cammas, 2003). Ces aménagements sont typiques des sols de l'intérieur des maisons. À Lattes, ils apparaissent $\mathrm{au} \mathrm{III}^{\mathrm{e}}$ siècle avant notre ère, et ils témoignent d'un entretien particulier de l'espace composé d'un remblai puis d'une alternance de ragréages et de surfaces piétinées avec des couvertures au sol. Ce type de préparation de sol a été rencontré de manière plus ponctuelle en contexte rural dans le nord de la France, comme dans des sols de petits celliers ou de caves de la fin de l'âge du Fer (sites de Compans, fouilles dirigées par Jean-Marc Séguier). Sur ces sites, localisés dans un contexte de luvisols sur loess, les sédiments utilisés sont décarbonatés et plus argileux. Dans ces aménagements, les ajouts végétaux restent rares.

La brique crue est largement utilisée à l'âge du Fer dans le sud de la France. À Lattes, ces éléments modulaires sont composés de limons d'inondation calcitiques, peu argileux et finement malaxés, comme ceux utilisés pour les ragréages. Il n'y a pas de dégraissant végétal, ni d'ajout de sables. Dans certaines briques, la présence de fissures verticales parallèles témoigne d'un moulage. L'homogénéité des caractères de la masse fine observée sur un grand nombre d'échantillons et celle des formes et des tailles des briques crues témoignent d'un savoir-faire élaboré, maîtrisé et standardisé dès le $\mathrm{V}^{\mathrm{e}}$ siècle avant notre ère.

Dans le nord de la France, la brique crue semble moins utilisée, et les élévations en brique crue d'époque romaine sont rares. À Mours (Val-d'Oise) [cf. p. 78], un four construit à l'aide de cette technique a été fouillé. L'analyse micromorphologique a montré une certaine variation dans la composition des briques [ill. 4 à 6] . Elles sont confectionnées à partir d'un mélange de sédiments qui proviennent principalement d'un luvisol, parfois mixés en proportion variable avec des sédiments provenant de dépôts carbonatés. La plupart des briques analysées présentent des traces de dégraissant végétal. Le degré de mélange varie de finement à très finement malaxé, et les fissures observées dans ces briques suggèrent qu'elles ont été moulées. Les analyses mettent en évidence des standards moins rigides que pour le site de Lattes. Sur le site de Mours, dans certaines briques, la présence de plages carbonatées microcristallines pose, encore une fois, la question de l'utilisation de la chaux comme stabilisant.

Un mode de préparation de la terre original est celui du pavage en terre crue d'une grande salle du château de Vincennes ${ }^{2}$. En lame mince, les pavés sont composés d'une superposition de lits millimétriques à centimétriques (Cammas, 1996a). Les lits correspondent à différents horizons pédologiques et dépôts géologiques régionaux, tels
2. Fouille dirigée par Peter MacIntyre de 1991 à 1996. 

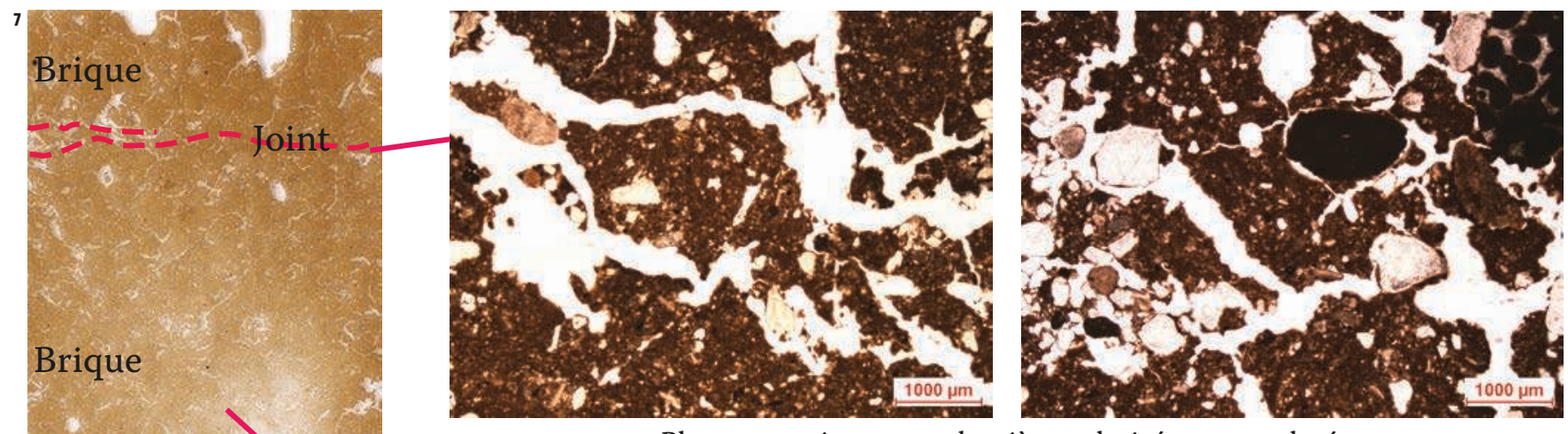

Photos au microscope, lumière polarisée non analysée

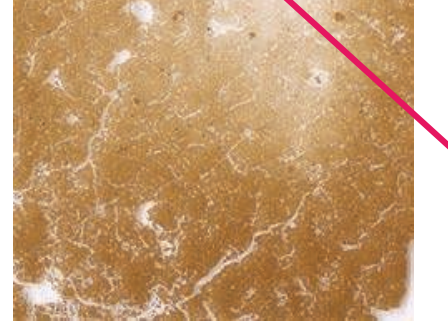

$\underset{0 \mathrm{~cm}}{\operatorname{Eg}}{ }_{1}$ Scan de lame mince

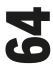

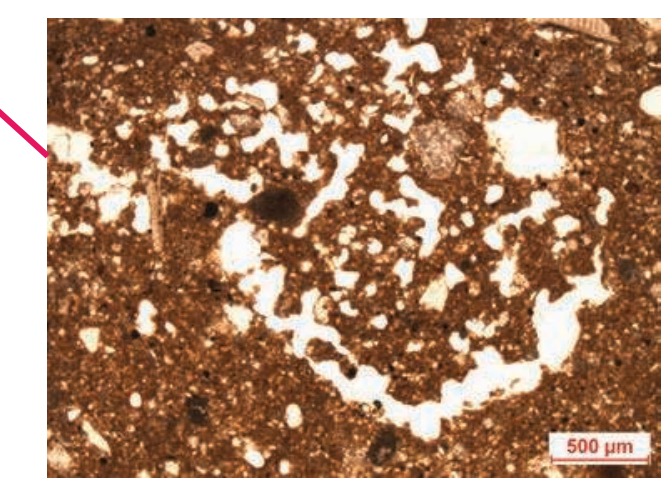

Photos au microscope,

lumière polarisée non analysée

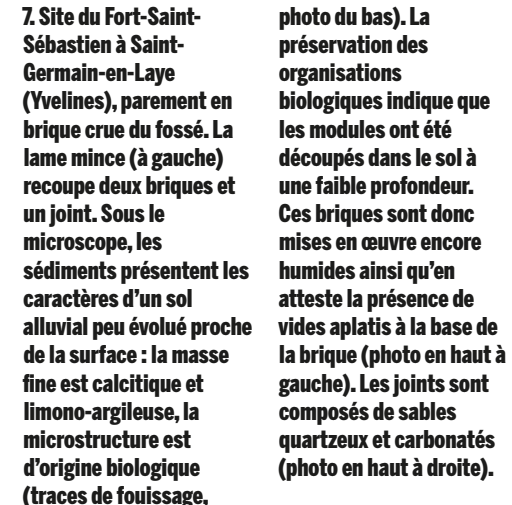

7. Site du Fort-Saint-
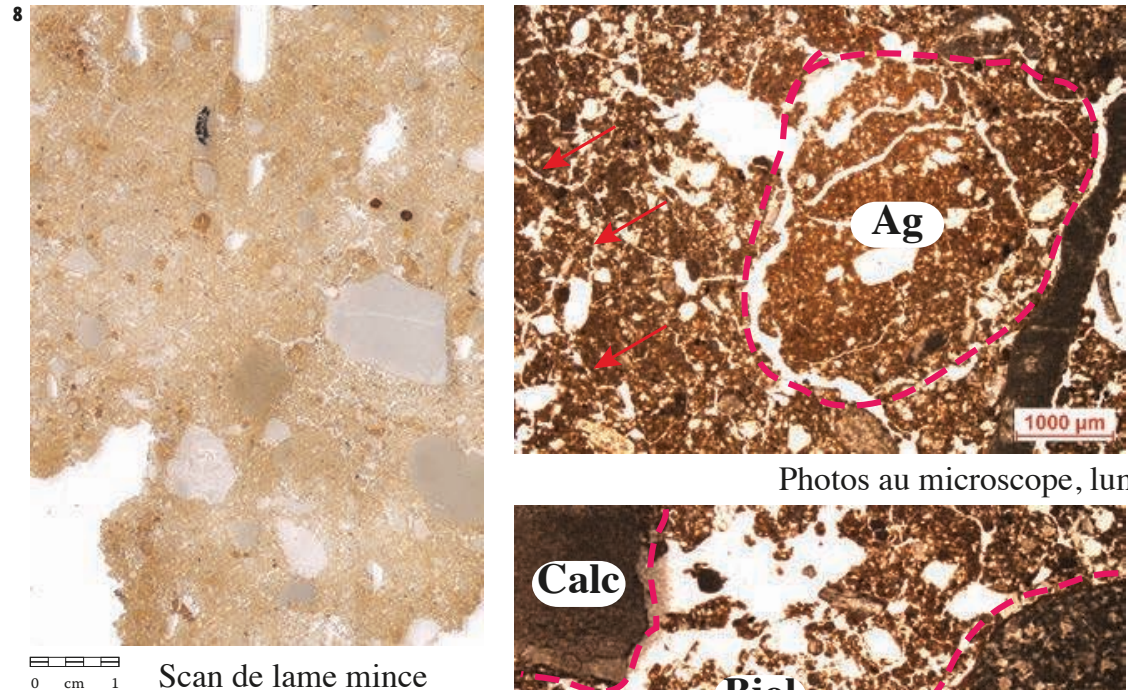

Photos au microscope, lumière polarisée non analysée

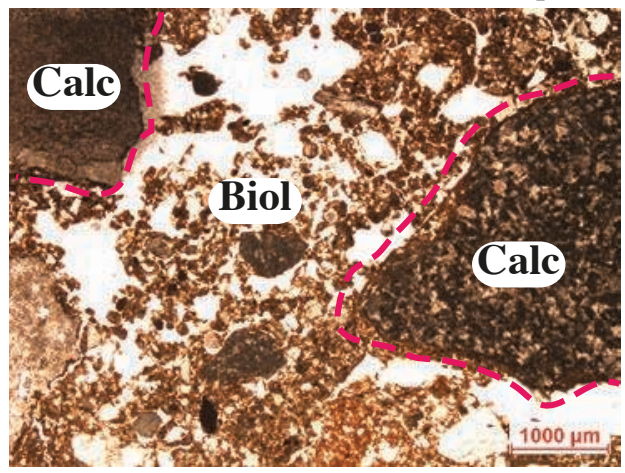

Photo au microscope, lumière polarisée non analysée

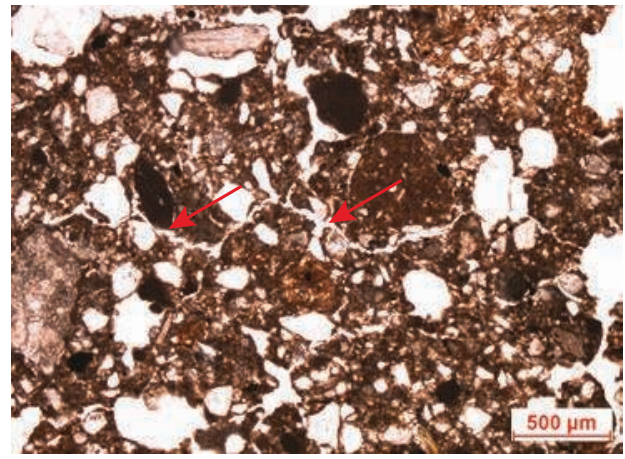

8.Site de Mouriès

(Bouches-du-Rhône,

gauche).La microstructure (éformation des agrégats élévation en terre massive. dans un état légèrement Sur la lame mince

(à gauche) et sous le microscope, des agrégats hétérogènes ( $\mathrm{Ag}$, photo en haut à gauche) et de nombreuses inclusions calcaires grossières et hétérométriques sont visibles (Calc, photo au microscope en bas à humide (fissures désignées par des flèches, photos en haut à gauche et en haut à droite).Des zones agrégées, qui résultent de l'activité biologique en place, peuvent être observées (Biol, photo en bas à gauche). Ces caractères sont typiques du pisé. 
qu'ils avaient été observés dans les coupes du site

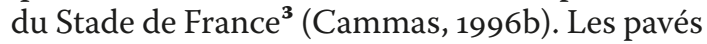
ont été confectionnés en empilant et compactant la terre, probablement dans de grands moules, l'absence d'effets de parois suggère la confection de grandes plaques découpées en éléments modulaires.

Un autre cas particulier est celui des parements en terre crue du grand fossé du site du Fort-SaintSébastien à Saint-Germain-en-Laye [cf. encadré p. 106] [ill. 7].

L'analyse micromorphologique montre que ces briques crues sont limono-argileuses, et que les sédiments ont conservé leur organisation typique de sol alluvial peu évolué : il s'agit de briques découpées dans des sols alluviaux à faible profondeur (Cammas, 2015).

Sur le terrain, il est parfois difficile d'identifier les modes de construction d'élévations en terre massive. Les comparaisons entre les analyses de matériaux actuels ou sub-actuels et les données archéologiques, confrontées aux données théoriques sur la construction en terre crue, ont permis de disposer de clés de détermination de la bauge et du pisé.

Le terme de bauge regroupe une grande diversité de techniques qui varient selon les périodes. Par exemple, à l'âge du Fer à Lattes, la bauge coffrée, le bourrage entre des parements en briques crues posées de chant, la bauge litée, les pains de terre sont utilisés simultanément (Roux et Cammas, 2010). L'observation en lame mince a permis de montrer qu'à Lattes, toutes ces techniques utilisent des matériaux similaires. Il s'agit de limons calcitiques beiges et de limons argileux plus jaunes, généralement grossièrement mélangés. Ils enrobent souvent des éléments grossiers tels que des mottes non homogénéisées ou des fragments de brique crue. Dans certains cas, le degré d'humidité observé en lame mince suggère très fortement l'utilisation de coffrage, hypothèse qui a pu être démontrée dans un cas grâce à un échantillonnage adapté (Roux et Cammas, 2007). Pour la bauge, la cohérence des sédiments est assurée par un mélange à l'état humide lors de la préparation des sédiments, et les éventuels coffrages servent alors à contenir les terres trop humides le temps du séchage.

Le pisé correspond à une technique bien définie et standardisée : les sédiments sont damés dans un coffrage au moment de la mise en place de la terre sur le mur. Lanalyse de cas archéologiques et ethnographiques montre que les sédiments peuvent provenir d'horizons de surface ou de sub-surface, souvent enrichis en graviers et grossièrement mélangés. Ils sont mis en place dans un état légèrement humide, état d'humidité qui autorise la compaction in situ dans les banchées [ill. 8].

Pour le Moyen Âge et le début de l'époque moderne, des élévations composées de levées séparées par des lits de bruyères - rempart de Sainte-Christied'Armagnac dans le Gers (Klein, 2003), maison de
Lézigan-Corbières dans l'Aude ${ }^{\mathbf{4}}$ - ont été observées. À Toulouse, cette technique est désignée comme «paret coffrée » sans que l'on puisse déterminer s'il s'agit de bauge ou de pisé (Loppe, 2012). À SainteChristie-d'Armagnac, l'analyse a montré que les sédiments utilisés sont décarbonatés et argileux. Ils sont mis en place et compactés dans un état peu humide qui semble se rapprocher de celui du pisé [ill. 9]. Il faut maintenant rechercher en lame mince la présence éventuelle de boules de terre dans ces couches de terre, argument qui permettrait d'étayer l'hypothèse de bauge (Loppe, 2012). Cet exemple illustre l'intérêt des investigations à l'échelle microscopique pour caractériser les chaînes opératoires. De plus, seule l'identification correcte des techniques, dans laquelle la micromorphologie joue un rôle important, permettra de connaître la date d'apparition du pisé ou de techniques plus locales telles que la « paret coffrée » au Moyen Âge et sa diffusion.

Le torchis est un mode de construction où la terre n'est pas porteuse. La terre est appliquée sur une armature végétale, le clayonnage, constituée en tressage plus ou moins serré et plus ou moins complexe (Duvernay, 2003). Dans les collections de référence, les torchis issus de sites ruraux de lâge du Fer dans le nord de la France sont confectionnés à partir des matériaux locaux tels que les dépôts d'inondations limono-sableux carbonatés peu évolués en contexte alluvial, ou encore les luvisols en contexte de plateaux, ainsi que le notait Théresia Duvernay (Duvernay, 2003). Dans la plupart des cas, les éléments étudiés ne présentent pas de dégraissant végétal. Les sédiments apparaissent finement malaxés et appliqués sur des baguettes circulaires. En revanche, les torchis d'époque romaine issus de sites parisiens comprennent d'abondantes inclusions grossières et un dégraissant végétal, probablement de la paille. Ces torchis portent l'empreinte d'une armature composée de baguettes circulaires, des empreintes rectangulaires témoignent de l'utilisation de lattes, ou de baguettes refendues pour constituer l'armature végétale (Cammas, 2000).

\section{Perspectives}

Les résultats des travaux menés en micromorphologie sur les matériaux de construction en terre crue présentés ci-dessus s'appuient sur des collections de référence larges, composées d'un grand nombre d'échantillons. Ces résultats soulignent que les matériaux utilisés sont essentiellement de provenance locale. Ils montrent que la micromorphologie permet de restituer les gestes des bâtisseurs et qu'elle est opérationnelle pour différencier les techniques. Ils documentent l'apparition, l'évolution et la diffusion de certaines techniques, comme l'apparition du ragréage à Lattes $\mathrm{au} \mathrm{IV}^{\mathrm{e}}$ siècle avant notre ère. Ces travaux devraient aussi permettre d'évacuer des idées toutes faites concernant ces mêmes matériaux. Lorsqu'elle est destinée à la construction en terre crue, la terre préparée ne comprend qu'une faible
3. Fouille dirigée par

Jean-Yves Dufour en 1995

et 1996.

4. Fouille dirigée en 2015 par Émilie Léal, Inrap.

\& 


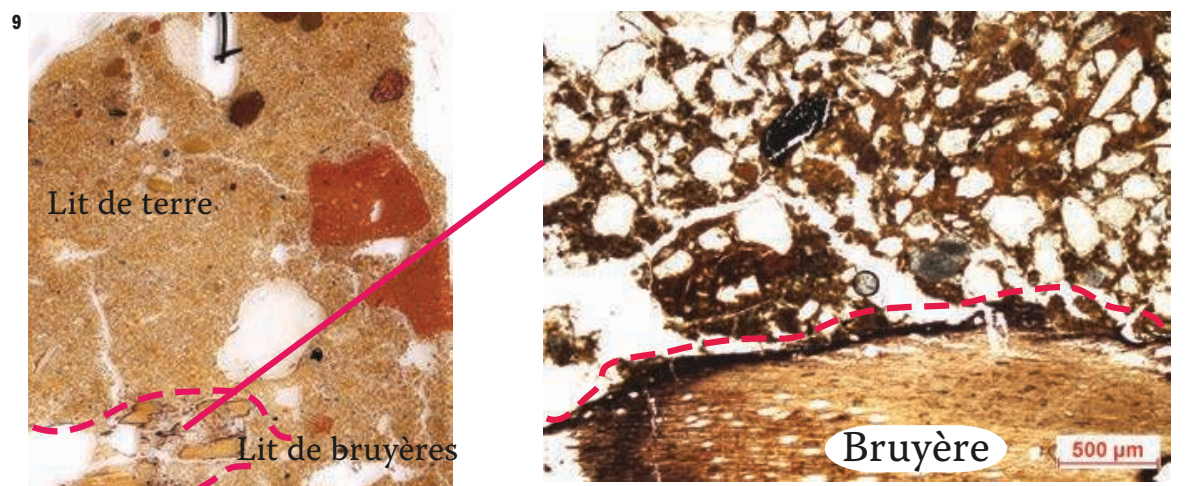

Photo au microscope,

lumière polarisée non analysée

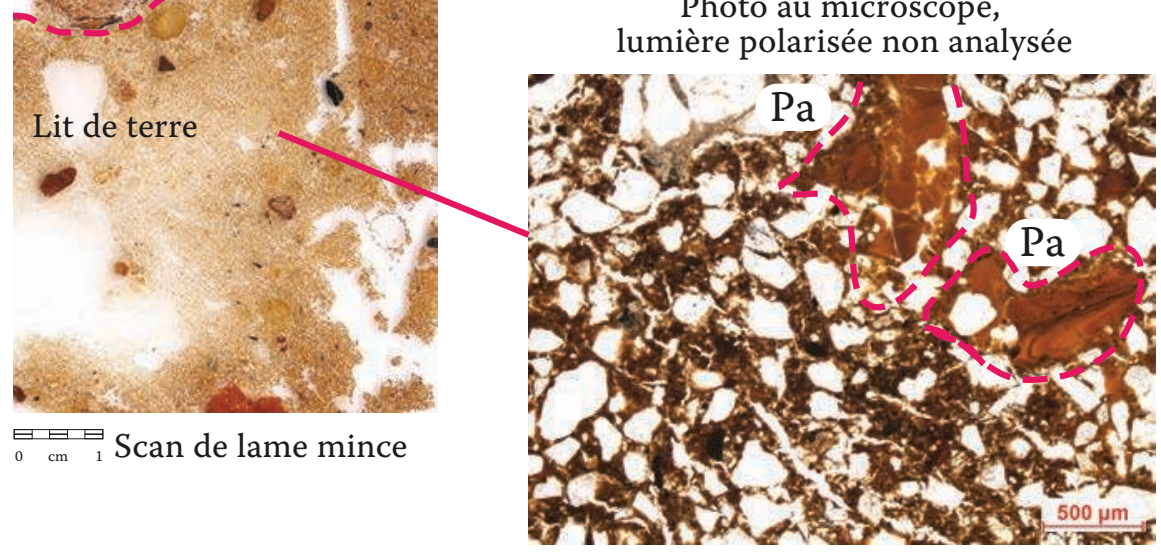

9. Rempart de SainteChristie-d'Armagnac, élévation en terre massive (bas Moyen Âge ?). La lame mince (à gauche) recoupe deux lits de terre et un lit de bruyère (agrandissement photo en haut à gauche). Les plages et les papules argileuses ( $\mathrm{Pa}$, photos en bas à droite et à gauche) marquent un mélange grossier des sédiments.
La microstructure fissurale témoigne d'une forte compaction (photos en haut et en bas à gauche). À l'échelle

microscopique, il est nécessaire de poursuivre les analyses avec un échantillonnage systématique sur la toute la hauteur d'un lit, et perpendiculaire à la paro du mur afin de préciser ce mode de construction.

Photos au microscope, lumière polarisée non analysée

proportion d'argiles. Les dégraissants végétaux et minéraux sont peu fréquents, surtout à l'âge du Fer, mais la texture peu argileuse des sédiments utilisés est plus stable, et les dégraissants ne semblent pas nécessaires. Les matériaux de construction en terre crue ne sont pas pauvres : non seulement le malaxage fin des sédiments est coûteux en temps, mais encore les parois en terre crue peuvent faire l'objet de traitements de surface soignés comportant des enduits peints dès le premier âge du Fer à Bourges (Cammas, 2008) ou des enduits de chaux à l'époque romaine (Cammas, 2000).

Au cours des dernières années, de nouvelles pistes de recherche concernant la construction en terre crue sont apparues du fait de découvertes originales comme, par exemple, les grandes plaques de torchis ou d'enduits talochés brûlés de l'âge du Bronze du site de Port-Montain (Noyen-sur-Seine, Seine-et-Marne). Ces matériaux rares sont encore à étudier et analyser. L'accent doit également être mis sur le Moyen Âge. Des techniques de construction typiques de cette période ont été identifiées, comme les élévations composées de levées séparées par des lits de bruyères. Ces restes d'architectures, remontant au Moyen Âge et encore en élévation, ont fait l'objet d'études archéologiques (Chazelles, 1997 ; Baudreu, 2003 ; Guyonnet, Catafau, 2003 ; Klein, 2003 ; Loppe, 2012). Ils méritent d'être analysés afin de caractériser ce matériau à l'échelle microscopique et d'apporter des précisions sur l'état d'humidité et le degré de tassement au moment de la construction. Le pisé reste peu représenté dans le corpus d'étude : est-ce du fait du déficit d'identification ou faute de questions posées à ce sujet? Cette question constitue également une piste de recherche afin de préciser la chronologie de son apparition et de sa diffusion.

Ces dernières années, des questions récurrentes se sont posées sur les sites des périodes protohistoriques et historiques. Parmi elles, la question du cru ou du cuit, par exemple, la nature des matériaux utilisés et le mode de confection des plaques foyères de l'âge du Fer. Les premiers résultats des analyses sur ces dernières, destinées à passer au feu lors de leur utilisation, montrent qu'elles sont confectionnées à partir de sédiments plus argileux, similaires à ceux utilisés pour fabriquer les briques cuites. Des questions se posent aussi de manière récurrente sur les enduits blanchâtres de l'âge du Fer (Cammas, 2008) et sur la possibilité de faire remonter l'utilisation de chaux au premier ou au second âge du Fer.

Un autre direction de la recherche concerne le regain d'intérêt actuel en faveur des techniques de construction en terre crue, telle que la bauge ou le pisé, adaptées à l'environnement et au climat. L'archéologie, surtout les travaux menés en micromorphologie, apparaissent alors comme une source d'information pour restituer les gestes des constructeurs, pour proposer des modes de construction à faible impact environnemental qui s'appuient sur ces techniques traditionnelles. 


\section{Références bibliographiques}

BAUDREU D., 2003, « Habitats et fortifications en terre crue d'époque médiévale dans le Midi de la France », in Chazelles C.-A. De, Klein A. (DIR.), 2003 Échanges transdisciplinaires sur les constructions en terre crue, vol. 1, Terre modelée, découpée ou coffrée, matériaux et modes de mise en œuvre, Actes de la table-ronde de Montpellier (17-18 novembre 2001), Montpellier, éditions de l'Espérou, p. 359-375.

CAmmas C., 1994, " Approche micromorphologique de la stratigraphie urbaine à Lattes : premiers résultats », in Lattara 7, Lattes, Association pour la recherche archéologique en Languedoc oriental (Aralo), 1994, p. 181-202.

Cammas C., 1996a, « Château de Vincennes, rapport géoarchéologique », in Fouilles du manoir de Vincennes, 1991-1996, DFS, rapport scientifique $\mathrm{n}^{\circ} 16$, tome 1, p. 197-219.

Cammas C., 1996b, «Étude géoarchéologique du site de Saint-Denis (Grand-Stade) », in MEYER O. (DIR.)

Dufour J.-Y., Marti F., CAMmas C., avec la contribution de PÉrU J.-J., GaborIEAU C. MADOURI I., Éléments archéologiques pour une histoire agraire de La Plaine Saint-Denis, SaintDenis (93), Cornillon Nord (Stade de France $=$ Grand-Stade), DFS, p. 69-117.

Cammas C., 1999, « Dynamique pédo-sédimentaire urbaine : modes de construction et d'occupation à Lattes au IV ${ }^{\mathrm{e}}$ S. av. n. è. », Lattara 12, Lattes, Aralo, p. 211-227.

Cammas C., 200o, Les matériaux de construction antiques à Paris: analyse micromorphologique d'éléments en torchis, mortier, plâtre et terre cuite (sites 410, 538, 547, 608, 634, 747, 609, 435, 345, 416, 558, 597), rapport d'analyse, Commission du Vieux Paris, novembre 2000

CAMmAS C., 2003, « L'architecture en terre crue à l'âge du Fer et à l'époque romaine : apports de la discrimination micromorphologique des modes de mise en œuvre », Chazelles C.-A. DE, Klein A. (DIR.), 2003, Échanges transdisciplinaires sur les constructions en terre crue, vol. 1, Terre modelée, découpée ou coffrée, matériaux et modes de mise en ouvre, Actes de la table-ronde de Montpellier (17-18 novembre 2001), Montpellier, éditions de l’Espérou, p. 33-53.

Cammas C., avec la contribution d'Allag C., 2008, "Approche micromorphologique du traitement de surface des murs sur les sites de Littré et de l'HôtelDieu au IV $\mathrm{IV}^{\mathrm{e}}$ s. et au ve s. BC à Bourges (Cher) », in Un complexe princier de l'âge du fer. L'habitat du promontoire de Bourges (VI $-I V^{e}$ s. av. J.-C.), BourgesTours, éd. de Bourges plus-Fédération pour l'édition de la Revue archéologique du Centre de la France, Supplément $\mathrm{n}^{\circ} 32$, p. 135-147.

CAmmas C., 2010, « Histoire microstratigraphique de structures d'habitat des îlots 27 et 1 de la ville de Lattara $(-375 /-475)$ », in JANIN T. (DIR.), Premières données sur le cinquième siècle avant notre ère dans la ville de Lattara, Lattara 21, tome 1, Lattes, Association pour le développement de l'archéologie en Languedoc-Roussillon (Adal), p. 289-324.

Cammas C., 2015, in Hurard S. (DIR.), Île-de-France, Yvelines, Saint-Germain-en-Laye, Fort SaintSébastien, rapport d'opération, Inrap-SRA Île-deFrance, vol. 3, études de mobilier, analyses botaniques et géoarchéologiques pour les périodes modernes, p. 421-432.

Cammas C. et Wattez J., 2009, « L'approche micromorphologique : méthode et applications aux stratigraphies archéologiques ", in La géologie : les sciences de la Terre, appliquées à l'Archéologie, Collection "Archéologiques", 2 éd. revue et augmentée, Paris, éditions Errance, p. 181-216.
Cammas C., Chazelles C.-A. de, 2013, «Archéologie des construction en terre crue dans les régions méditerranéennes, problématiques, moyens d'investigation et résultats », Conférence inaugurale de la session thématique 4, de la production à la diffusion, matériaux en terre crue ou cuite : de la matière première à la manière de façonner construire et distribuer, XIX ${ }^{e}$ Colloque d'Archéométrie du Groupement des Méthodes Pluridisciplinaires en Archéologie (Caen, 22-26 avril 2013), à paraître.

Chazelles C.-A. de, 1997, Les maisons en terre de la Gaule méridionale, Montagnac, Monique Mergoil, $231 \mathrm{p}$.

Courty M.-A., Golberg P. et Macphail R.I., 1989, Soils and micromorphology in archaeology, Cambridge, Cambridge University Press, 340 p.

DUVERnAY T., 2003, « La construction en terre crue: potentiel des restes en position secondaire. Le cas d'un site rural du Bassin parisien (opérations d'archéologie préventive de Ville-Saint-Jacques / Varennes-sur-Seine, Seine-et-Marne 77) », in Chazelles C.-A. DE, Klein A. (DIR.), 2003, Échanges transdisciplinaires sur les constructions en terre crue, vol. 1, Terre modelée, découpée ou coffrée matériaux et modes de mise en ceuvre, Actes de la table-ronde de Montpellier (17-18 novembre 2001), Montpellier, éditions de l'Espérou, p. 55-71.

FEDOROFF N., 1979, « Organisation du sol à l'échelle microscopique », in BONNEAU M., SOUCHIER B. (ÉD.), Constituants et propriétés du sol, Pédologie, vol. 2, Paris, Masson, p. 251-265.

Fedoroff N., Courty M.-A., 1994, « Organisation du sol à l'échelle microscopique », in BONNEAU M., SOUCHIER B. (ÉD.), Constituants et propriétés $d u$ sol, Pédologie, vol. 2, $2^{\mathrm{e}}$ édition, Paris, Masson, p. 349-375.

Friesem D., Karkanas P., Tsartsidou G., ShahackGross R., 2014, « Sedimentary processes involved in mud brick degradation in temperate environment : a micromorphological approach in an ethnoarchaeological context in northern Greece », Journal of Archaeological Science, 41, p. 556-567.

Gé T., Courty M.-A., Matthews W., Wattez J., 1993 «Sedimentary Formation Processes of Occupation Surfaces ", in Goldberg P., Nash T. D., Petraglia M. D. (ÉD.), Formation Processes in Archaeological Context, Madison, Wisconsin, Prehistory Press, «Monographs in World Archaeology », 17, p. 149-163.

Goldberg P., MacPhail R. I., 2006, Practical and theorical geoarchaeology, Oxford, Blackwell Publishing, 454 p.

GoOdMAN-Elgar M., 2008, « The devolution of mudbrick: ethnoarchaeology of abandoned earthen dwellings in the Bolivian Andes ", Journal of Archaeological Science, 35, p. 3057-3072.

GUYONNET F., CATAFAU A., 2003, "La construction urbaine en terre au XIII ${ }^{e} s$. : l'exemple de la rue de l'Anguille (Perpignan) », in Chazelles C.-A. DE, KLEIN A. (DIR.), 2003, Échanges transdisciplinaires sur les constructions en terre crue, vol. 1, Terre modelée, découpée ou coffrée, matériaux et modes de mise en ouvre, Actes de la table-ronde de Montpellier (17-18 novembre 2001), Montpellier, éditions de l'Espérou, p. 389-411.

KLEIN A., 2003, « La construction en terre crue par couches continues, en Midi-Pyrénées. $\mathrm{XVI}^{\mathrm{e}}-\mathrm{XX}^{\mathrm{e}} \mathrm{S}$. Contribution à l'identification des techniques ", in Chazelles C.-A. DE, Klein A. (DIR.), 2003 Échanges transdisciplinaires sur les constructions en terre crue, vol. 1, Terre modelée, découpée ou coffrée, matériaux et modes de mise en ouvre, Actes de la table-ronde de Montpellier (17-18 novembre 2001), Montpellier, éditions de l'Espérou, p. 417-437.
Loppe F., 2012, Construire en terre à Toulouse en 13541355, Service Connaissance du Patrimoine ; DCAV - Région Midi-Pyrénées, http://patrimoines. midipyrenees.fr/fileadmin/Patrimoines-editorial/ Les_thematiques/Architecture/Terre_crue/TC ConstruireToulouseOpt.pdf

Matthews W., 1995, « Micromorphological characterisation of occupation deposits and microstratigraphic sequences at Abu Salabikh, Southern Iraq ", in BARHAM A. J., MACPHAIL R. I. (ÉD.), Archaeological Sediments and Soils: Analysis, Interpretation and Management, Londres, Institute of Archaeology, University College, p. 41-76.

Onfray, M., 2014. « La question de l'utilisation du torchis dans l'aménagement des enceintes néolithiques du Centre-Ouest de la France : exemples comparés des encientes de ChampDurand à Nieuil-sur-l'Autise (Vendée) et de Bellevue à Chenommet (Charente) ", in Joussaume R. LARGE J.-M. et al. (ÉD.), enceintes néolithiques de l'Ouest de la France de la Seine à la Gironde, Actes du Colloque CrabeNéo, Chauvigny, Association des Publications Chauvinoises (Mém. XLVIII), p. 322-334.

Rosen A. M., 1993, « Microartifacts as a Reflection of Cultural Factors in Site Formation », in GOLDBERG P., Nash T. D., Petraglia M. D. (ÉD.), Formation Processes in Archaeological Context, Madison, Wisconsin, Prehistory Press, « Monographs in World Archaeology », 17, p. 141-148

Roux J.-C., CAMmas C., 2007, « La bauge coffrée : appréhension d'un mode de construction inédit dans la ville protohistorique de Lattes, Hérault (deuxième quart du IV ${ }^{e}$ s. av. n. è.) », in GuIllaud H., Chazelles C.-A. De, Klein A. (Dir.), Les constructions en terre massive : pisé et bauge, échanges transdisciplinaires sur les constructions en terre crue 2, Actes de la table-ronde de Villefontain (Isère, 28-29 mai 2005), Montpellier, éd. de l'Espérou, p. 87-98.

Roux J.-C., Cammas C., 2010, « Les techniques constructives en bauge dans l'architecture protohistorique de Lattara, Lattes, Hérault (milieu $\mathrm{V}^{\mathrm{e}}$-milieu IV ${ }^{\mathrm{e}}$ s. av. n. è.) », in JANIN T. (DIR.), Premières données sur le cinquième siècle avant notre ère dans la ville de Lattara, Lattara, 21, Association pour le Développement de l'Archéologie en Languedoc, p. 219-288.

WATTEZ J., 2003, « Caractérisation micromorphologique des matériaux façonnés en terre cuite dans les habitats du Sud de la France : l'exemple des sites Jacques Cour (Montpellier Hérault), du Jas del Biau (Millau, Aveyron) et de la Capoulière (Mauguio, Hérault) », in CHAZELles C.-A. DE, KLEIN A. (DIR.), Echanges transdisciplinaires sur les constructions en terre crue, Montpellier, Éditions de l'Espérou, p. 21-31.

WATTEZ J., 2009, «Enregistrement sédimentaire de l'usage de la terre crue dans les établissements néolithiques du sud de la France : le cas des sites du Néolithique Final de La Capoulière 2 et du Mas de Vignoles IV », in BeECHING A. ET SÉnÉPART I. (DIR.), De la maison au village: l'habitat néolithique dans le Sud de la France et le Nord-Ouest méditerranéen, Actes de la table ronde des 23 et 24 mai 2003 (Marseille, Musée d'histoire de la ville de Marseille), Paris, Société Préhistorique Française, p. 199-218. 


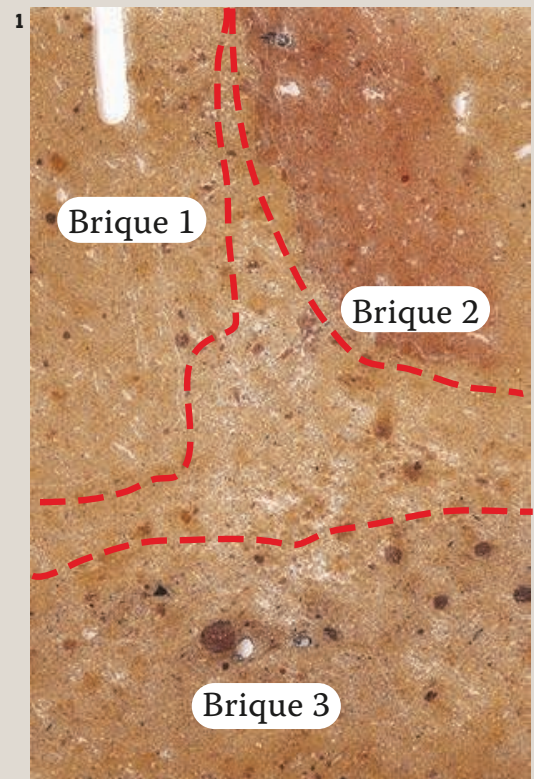

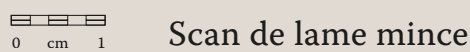
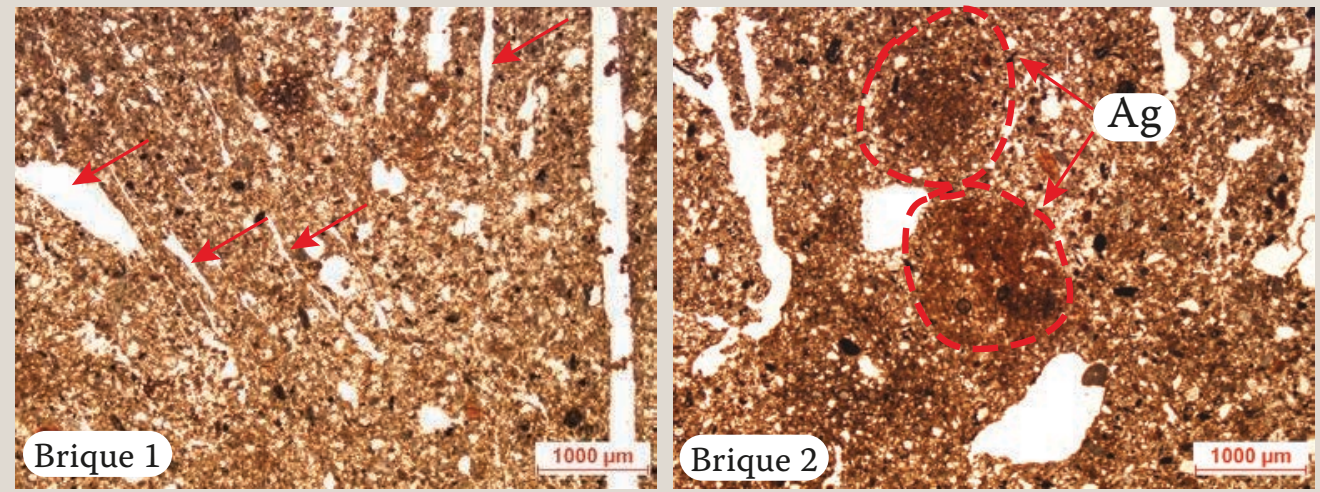

Photos au microscope, lumière polarisée non analysée

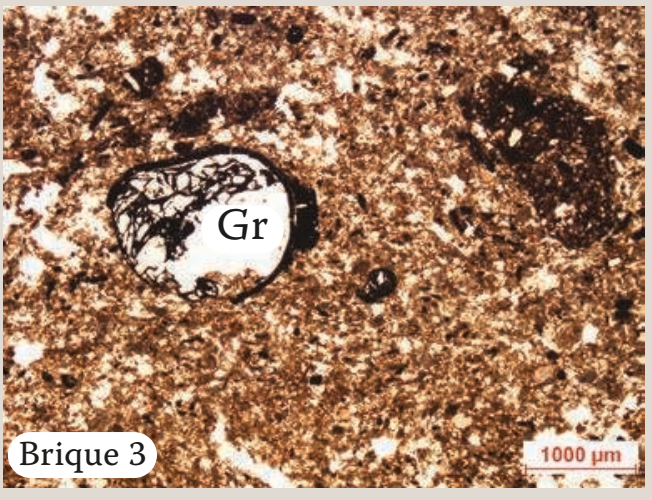

Photo au microscope,

lumière polarisée non analysée
1. Lame mince dans trois briques crues maurétaniennes (scan à gauche). Sous le microscope, la brique 1 présente des fantômes végétaux qui témoignent de la présence d'un dégraissant végétal (photo en haut à gauche). Dans la brique 2, des agrégats résiduels marquent un malaxage modéré (photo en haut à droite). La brique 3 présente des graines carbonisées (photo en bas à gauche).

\section{Étude des} matériaux de construction en terre crue des sites antiques de Rirha (Maroc)

\section{Cécilia Cammas}

Inrap, UMR 5140, "Archéologie des sociétés méditerranéennes "

\section{Jean-Claude Roux}

ministère de la Culture, UMR 5140, "Archéologie des sociétés méditerranéennes »
Les sites successifs de Rirha (Sidi Slimane, Maroc) ${ }^{1}$ sont installés dans la riche plaine agricole du Gharb. La zone archéologique, en domaine alluvial, est actuellement enserrée dans un méandre récent du Beht. La fouille porte sur un tell dont l'occupation débute à l'âge du Fer, et qui est vraisemblablement agrandi durant l'époque romaine, puis réoccupé à l'époque médiévale entre le $x^{\mathrm{e}}$ et le $\mathrm{xrv}^{\mathrm{e}}$ siècle. Pour la période maurétanienne, la terre crue-plus particulièrement la brique - est exclusivement privilégiée pour la construction. Ce site of frait l'occasion d'étudier ce matériau, et un programme de recherche interdisciplinaire sur l'emploi de la terre crue en architecture ${ }^{2}$ (archéologique et micromorphologique) intégré au programme de fouille a été défini. Ce projet visait, pour la partie de terrain (J.-C. Roux), à identifier les élévations en terre crue et la mise en œuvre des briques dans les bâtiments (sous-bassements, élévations, chaînages). Une typologie macroscopique des briques, fondée sur le format, les dimensions, la texture, la couleur des briques et les inclusions a été élaborée, puis chaque type de brique a été échantillonné pour l'analyse micromorphologique, ainsi que les joints et les enduits. Des prélèvements comprenant deux briques accolées, ou deux briques superposées, ont été réalisés afin de caractériser briques et joints. L'analyse des briques, coupées selon différents plans, avait pour but d'identifier des caractères liés au tassement dans un moule, ou encore les aménagements du sol au moment du moulage ou du séchage (Roux et Cammas, sous presse). L'objectif de l'analyse micromorphologique était de compléter la caractérisation de la terre utilisée, ainsi que les traces de façonnage, difficilement perceptibles à l'œil nu. La démarche présentée ici peut être transposée pour l'étude de tout lot d'éléments modulaires en terre crue. Pour l'époque maurétanienne, dix briques ont été analysées. Les résultats montrent que les sédiments ont une texture assez variée, et qu'ils proviennent de différents dépôts alluviaux à composante éolienne plus ou moins marquée. Sur le terrain et à l'échelle microscopique, des inclusions anthropiques diverses ont été observées : des charbons, des fragments d'os à différents degrés de combustion, des matériaux de construction (matériaux malaxés chauffés ou non), des granules de céramique. Leur faible proportion suggère que leur présence est aléatoire ; néanmoins, ils indiquent que les premières étapes de la chaîne opératoire (prélèvement, malaxage) se déroulaient à proximité des zones anthropisées. La dimension et l'abondance des résidus végétaux attestent, quant à elles, 


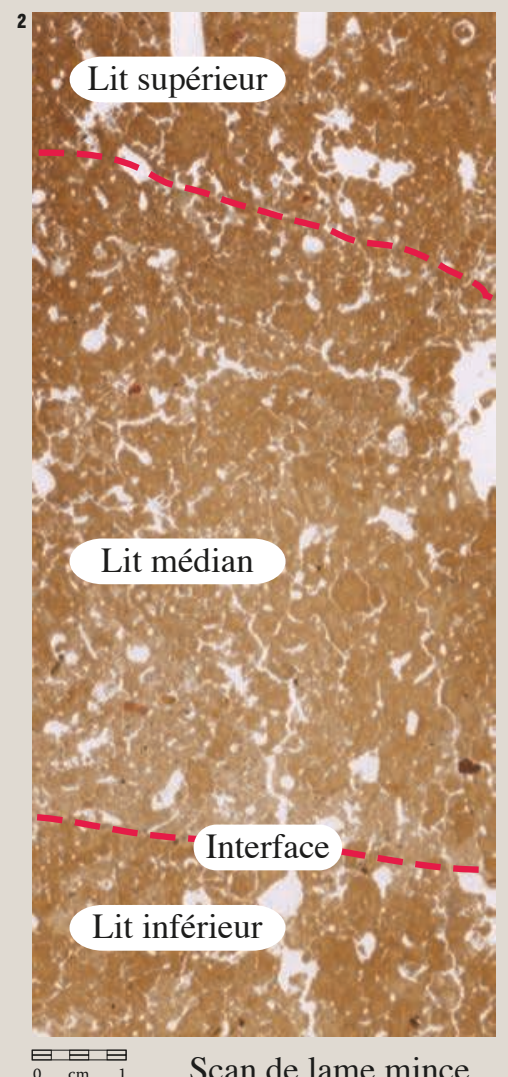

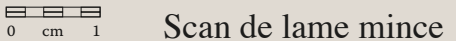

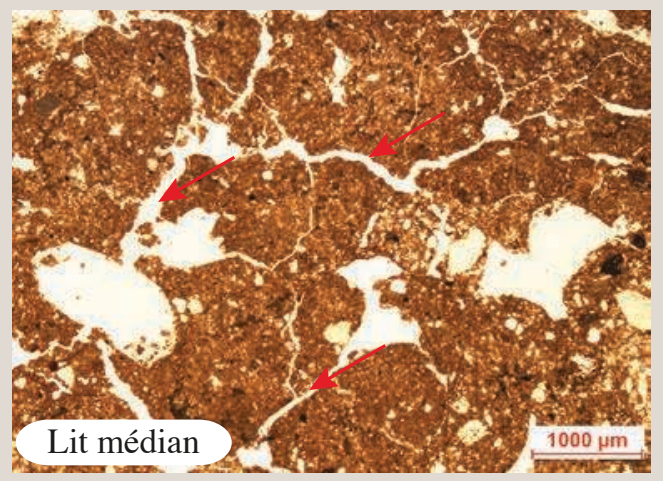

Photo au microscope,

lumière polarisée non analysée
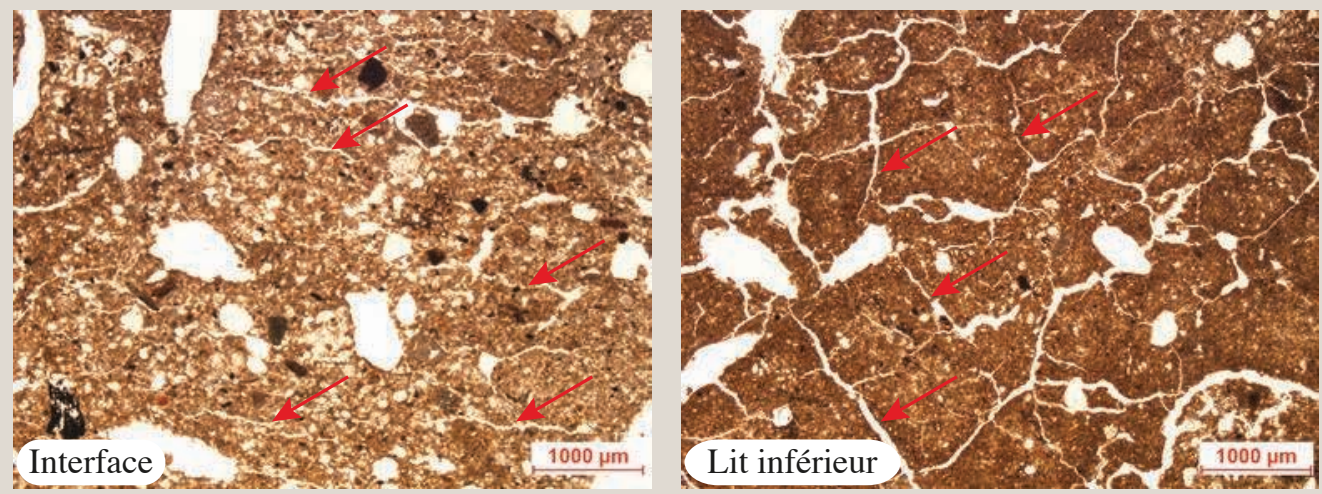

Photos au microscope, lumière polarisée non analysée
2. Lame mince au cœur du mur en terre massive (scan à gauche). Sous le microscope, le réseau de fissures résulte de la déformation et de l'accommodation des agrégats entre eux (photo en haut à gauche et en bas à droite). L'interface entre les lits est marquée par des fissures subhorizontales, l'orientation des fissures marque une surface de tassement des sédiments (photo en bas à gauche). d'un ajout volontaire, et les analyses archéobotaniques menées par Emmanuelle Bonnaire indiquent que des déchets végétaux divers, issus de différentes étapes de traitement des végétaux, étaient incorporés aux briques (Bonnaire, sous presse). L'abondance des agrégats résiduels est inversement proportionnelle au degré de malaxage, ici la variation de la composition d'une brique à l'autre montre que les éléments d'une même élévation peuvent présenter des degrés de malaxage variés.

L'aplatissement des faces supérieures ou inférieures de certaines briques témoigne du tassement de la terre dans les moules, et les empreintes végétales résultent de l'utilisation de bouchons de paille pour tasser la terre, ou de lits de végétaux épandus au sol au moment du moulage. Fn lame mince, l'identification d'agrégats roulés par le piétinement entre les assises suggère que le bâtisseur devait se placer sur le mur au moment de la construction. Les résultats de ces travaux montrent l'absence de standardisation en ce qui concerne la terre utilisée et le degré de malaxage. La juxtaposition de briques différentes au sein d'une même élévation [ill. 1] suggère des normes souples mais précises pour la confection des briques, plutôt que des savoir-faire différents. Les mises en cuvre, sur un, deux ou trois rangs de briques disposées en parpaing, en boutisse ou en panneresse, se composent d'éléments divers selon l'épaisseur du mur ; des formats se distinguent particulièrement : $34-35 \mathrm{~cm}$ x $55-57 \mathrm{~cm}$ et $36-38 \mathrm{~cm} \times 54-58 \mathrm{~cm}$, avec un module plus constant de $34-36 \mathrm{~cm} \times 49-52 \mathrm{~cm}$. La réutilisation de briques crues d'un état à l'autre a pu être mise en évidence. Dans la domus en cours de fouille, la découverte d'un mur en terre massive du $\mathrm{rr}^{\mathrm{e}}$ siècle, dont le mode de construction était énigmatique, a été l'occasion de tester les clés de détermination élaborées à l'échelle microscopique entre la bauge et le pisé. Les résultats de l'analyse montrent que les sédiments, à dominante limono-argileuse, présentent d'abondantes inclusions anthropiques grossières, comme pour les briques maurétaniennes, mais ici des agrégats de chaux sont également présents. Les sédiments ne sont pas malaxés, et la microstructure indique que les agrégats ont été entassés dans un état moyennement humide, ce qui permet le tassement mécanique. Ainsi, les caractères micromorphologiques suggéraient qu'il s'agissait d'un mur en pisé [ill. 2]. La reprise des fouilles, l'année suivante, a permis de trouver les trous de clés supportant le coffrage, ce qui confirmait ainsi l'utilisation de la technique du pisé. À travers ces exemples, les apports de la micromorphologie à la connaissance de la chaîne opératoire se dessinent. Plus particulièrement, le lieu de provenance des terres, les ajouts au moment de la préparation du matériau en terre à bâtir (addition d'eau, de végétaux) et son degré de plasticité au moment de la confection (briques) et à la pose (pisé, liants, enduits...) sont des informations originales que la micromorphologie peut apporter.

Références bibliographiques

BonNAIRE E., sous presse, « Étude archéobotanique », in Callegarin L., Kbiri Alaoui M. et ICHKhaKh A. (DIR.), Le site de Rirha (Sidi Slimane, Maroc). Les occupations antique et médiévale, Madrid, Mélanges de la Casa de Velazquez.

Roux J.-C., CAMmas C., sous presse, « L'architecture en terre crue maurétanienne », in CALlegarin L., KBIR AlaOui M. Ет ICHKHAKH A. (DIR.), Le site de Rirha (Sidi Slimane, Maroc). Les occupations antique et médiévale, Madrid, Mélanges de la Casa de Velazquez. 